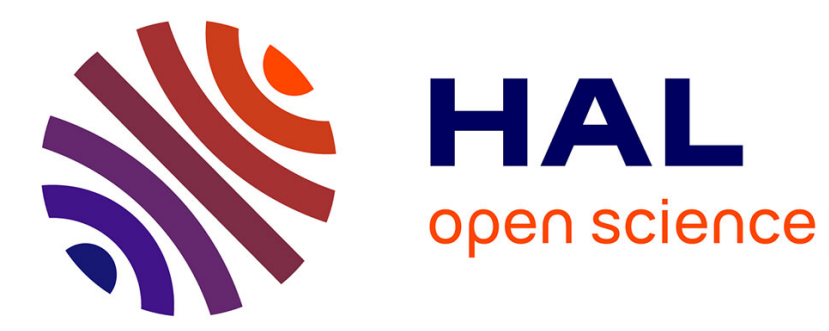

\title{
Autocontinuity and convergence theorems for the Choquet integral
}

\author{
Yann Rébillé
}

\section{To cite this version:}

Yann Rébillé. Autocontinuity and convergence theorems for the Choquet integral. 2009. hal00422562

\section{HAL Id: hal-00422562 \\ https://hal.science/hal-00422562}

Preprint submitted on 7 Oct 2009

HAL is a multi-disciplinary open access archive for the deposit and dissemination of scientific research documents, whether they are published or not. The documents may come from teaching and research institutions in France or abroad, or from public or private research centers.
L'archive ouverte pluridisciplinaire HAL, est destinée au dépôt et à la diffusion de documents scientifiques de niveau recherche, publiés ou non, émanant des établissements d'enseignement et de recherche français ou étrangers, des laboratoires publics ou privés. 


\title{
Autocontinuity and convergence theorems for the Choquet integral
}

\author{
Yann REBILLÉ (*)
}

$2009 / 26$

$\left({ }^{*}\right)$ LEMNA, Université de Nantes

INSTITUT

d'ECONOMIE

et de MANAGEMENT

de NANTES - IAE
EA 4272

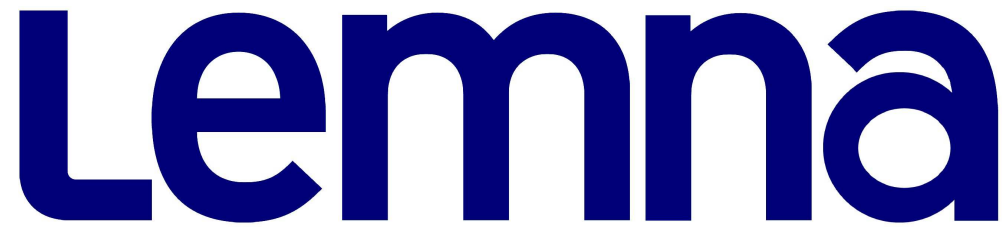




\title{
Autocontinuity and convergence theorems for the Choquet integral
}

\author{
Yann Rébillé * \\ LEMna, Institute of Economics and Management of Nantes-IAE, \\ chemin la Censive du Tertre, BP 62232, 44322 Nantes Cedex 3, France.
}

\begin{abstract}
Our aim is to provide some convergence theorems for the Choquet integral with respect to various notions of convergence. For instance, the dominated convergence theorem for almost uniform convergence is related to autocontinuous set functions. Autocontinuity can also be related to convergence in measure, strict convergence or mean convergence. Whereas the monotone convergence theorem for almost uniform convergence is related to monotone autocontinuity, a weaker version than autocontinuity.
\end{abstract}

Keywords: Choquet integral, null-additive set functions, autocontinuity.

\section{Introduction}

Convergence theorem for sequences of measurable functions play a central role in classical measure theory. Convergence in mean or in measure for instance are traditionally related to the sigma-additivity of a measure, a continuity condition. Generalizations of classical measure theory can be pursued in the direction of non-additive set functions such as fuzzy measures. A key property of additivity is null additivity (Pap [1]), whereas sigma-additivity can be associated to strong continuity. An intermediate notion introduced by Wang [2] is the one of autocontinuity. Autocontinuity guarantees null-additivity and contains some regularity conditions related to continuity but does not necessarily entail strong continuity. Wang's article explores the validity of classical convergence theorem for sequences of measurable functions in term of their fuzzy integrals. A similar approach is

\footnotetext{
*Yann.Rebille@univ-nantes.fr
} 
pursued with respect to non-additive integration in term of Choquet integrals, a generalization of the standard Lebesgue integral ([3]). Classical theorems such as the monotone or the dominated convergence theorem are established therein. Our aim is to identify which properties a set function might possess when we consider different types of convergence: - convergence in measure $(\mu)$, almost everywhere convergence (a.e.), almost uniform (a.u.) convergence or convergence in mean $(m)$, and different modes of convergence: -monotone, dominated. As it turns out, dominated convergence theorems with a.u. convergence are unsurprisingly related to autocontinuity. Still, autocontinuity can also be characterized through $\mu$ convergence, $m$ convergence and in a more direct way through strict convergence. Whereas dominated convergence theorems with a.e. convergence are related to strong continuity. As for the monotone convergence theorems with a.u. convergence, they are related to a weaker version of autocontinuity, namely: autocontinuity from below or above. These properties trace back to [2].

Next section introduces the relevant material for our study. In section 3 we present various types of convergence theorems whether we consider a.u., a.e. or $\mu$ convergence. Section 4 restates our results by duality. Finally, we illustrate through examples that autocontinuity, order continuity and monotone autocontinuity are independent properties.

\section{Preliminaries}

Let $(\Omega, \mathcal{A})$ be a measurable space and $\mu$ a set function, $\mu: \mathcal{A} \rightarrow \mathbb{R}$. From now on we will assume that $\mu(\emptyset)=0$.

$\mu$ is monotone if for all $A, B \in \mathcal{A}, \mu(A) \leq \mu(B)$ whenever $A \subset B$.

We define the conjugate set function $\bar{\mu}$ by $\bar{\mu}(A)=\mu(\Omega)-\mu\left(A^{c}\right)$.

A sequence $\left\{f_{n}\right\}_{n}$ of finite-valued measurable functions converges almost everywhere to $f$ if there is a set $E$ with $\mu(E)=0$ such that the sequence $\left\{f_{n} \mathbb{1}_{E^{c}}\right\}_{n}$ converges to $f \mathbb{1}_{E^{c}}$. The sequence $\left\{f_{n}\right\}_{n}$ converges almost uniformly to $f$ if for all $\epsilon>0$ there is a set $E_{\epsilon}$ with $\mu\left(E_{\epsilon}\right)<\epsilon$ such that the sequence $\left\{f_{n} \mathbb{1}_{E_{\epsilon}^{c}}\right\}_{n}$ converges uniformly to $f \mathbb{1}_{E_{\epsilon}^{c}}$. We shall write as usual $f_{n} \stackrel{\text { a.e. }}{\longrightarrow} f$ and $f_{n} \stackrel{a . u .}{\longrightarrow} f$ respectively, and $\downarrow, \uparrow$ if the convergence is monotonically non-increasing, non-decreasing.

Let $f$ be a measurable bounded function and $\mu$ a monotone set function. The Choquet integral of $f([4])$ with respect to $\mu$ is given by

$$
\int f d \mu=\int_{0}^{\infty} \mu(\{f \geq t\}) d t+\int_{-\infty}^{0} \mu(\{f \geq t\})-\mu(\Omega) d t
$$

In the integrand, the large inequalities may be replaced by strict ones since $\mu$ is monotone. The computation with respect to the conjugate set function is given by $\int f d \bar{\mu}=-\int-f d \mu$.

We will focus our study to bounded measurable functions, a set large enough to deal with usual applications where the existence of the Choquet integral is always guaranteed. We denote by $B_{\infty}(\Omega)\left(B_{\infty}(\Omega)^{+}\right)$the set of (non-negative) bounded and measurable functions defined on $\Omega$. 
Two functions $f, g$ are said to be comonotonic or compatible if for all $\omega, \omega^{\prime} \in$ $\Omega, f(\omega)>f\left(\omega^{\prime}\right) \Rightarrow g(\omega) \geq g\left(\omega^{\prime}\right)([5,6,7])$. In that case,

$$
\int f+g d \mu=\int f d \mu+\int g d \mu \text {. }
$$

Let us recall three properties that link continuity condition of a set function to the convergence of a sequence of functions and their Choquet integrals.

Property 2.1 Let $\mu$ be a set function. Then, $\mu$ is monotone if and only if for all $\left\{f_{n}\right\}_{n} \cup\{f\} \subset B_{\infty}(\Omega)$ if $f_{n} \downarrow_{u \text {. }}\left(\uparrow_{u}.\right) f$ then $^{1} \int f_{n} d \mu \downarrow(\uparrow) \int f d \mu$.

In order to extend Property 2.1 to everywhere convergence some further continuity conditions on the set function are introduced.

$\mu$ is said to be order continuous if $\mu\left(A_{n}\right) \downarrow 0$ whenever $A_{n} \downarrow \emptyset$.

$\mu$ is be continuous from above if for all $A_{n} \downarrow A$ then $\mu\left(A_{n}\right) \downarrow \mu(A)$. By definition a continuous from above set function is monotone (take $A_{1}=A, A_{n}=B$ for $n \geq 2$ ) and order continuous. $\mu$ is said to be continuous from below if for all $A_{n} \uparrow A$ then $\mu\left(A_{n}\right) \uparrow \mu(A)$.

Property 2.2 Let $\mu$ be a set function. Then, $\mu$ is monotone and order continuous if and only if for all $\left\{f_{n}\right\}_{n} \subset B_{\infty}(\Omega), \alpha \in \mathbb{R}$ if $f_{n} \downarrow_{\text {e. }} \alpha . \mathbb{1}_{\Omega}$ then $\int f_{n} d \mu \downarrow \alpha \mu(\Omega)$.

Property 2.3 Let $\mu$ be a monotone set function. Then, $\mu$ is monotone continuous from above (below) if and only if for all $\left\{f_{n}\right\}_{n} \cup\{f\} \subset B_{\infty}(\Omega)$ if $f_{n} \downarrow_{\text {e. }}\left(\uparrow_{\text {e. }}\right) f$ then $\int f_{n} d \mu \downarrow(\uparrow) \int f d \mu$.

Our aim is to extend Properties 2.1 and 2.3 to a.u. and a.e. convergence. To deal with these types of convergence we must recall some facts about nulladditivity.

A set $E$ is said to be null if for all $F, \mu(E \cup F)=\mu(F)$.

$\mu$ is said to be null-additive if $\mu(E \cup F)=\mu(F)$ for all $F$ whenever $\mu(E)=0$, or equivalently $\mu(F)=\mu(F \backslash E)$ for all $F$ whenever $\mu(E)=0$.

This way a set function $\mu$ is null-additive if and only if for all $E, E$ is null as soon as $\mu(E)=0$. The following proposition characterizes null-additivity in terms of Choquet integrals $([1,8])$,

Proposition 2.1 Let $\mu$ be a monotone set function. Then, $\mu$ is null-additive

$$
\Longleftrightarrow
$$

if $\mu(E)=0$ then for all $f \in B_{\infty}(\Omega)^{+}, \int f d \mu=\int f \mathbb{1}_{E^{c}} d \mu$

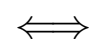

if $\mu(E)=0$ then for all $f, g \in B_{\infty}(\Omega)^{+}, f \mathbb{1}_{E^{c}}=g \mathbb{1}_{E^{c}} \Rightarrow \int f d \mu=\int g d \mu$.

\footnotetext{
${ }^{1}(i f)$. We can observe that the Choquet integral is always defined for indicator functions,
} $\int \mathbb{1}_{C} d \mu=\mu(C)$ for all $C \in \mathcal{A}$. Thus $A \subset B \Longleftrightarrow \mathbb{1}_{A} \leq \mathbb{1}_{B} \Rightarrow \mu(A) \leq \mu(B)$, so $\mu$ is monotone. 
Proof: For the first equivalence, $(\Leftarrow)$ is straightforward with $f=\mathbb{1}_{F}+\mathbb{1}_{E}$ with $E \cap F=\emptyset$.

$(\Rightarrow)$ Let $t>0$. Then,

$$
\{f \geq t\}=\{f \geq t\} \cap E+\{f \geq t\} \cap E^{c}=\left\{f \mathbb{1}_{E} \geq t\right\}+\left\{f \mathbb{1}_{E^{c}} \geq t\right\} .
$$

Now since $\mu(E)=0$ we have also have $\mu\left(\left\{f \mathbb{1}_{E} \geq t\right\}\right)=0$ for all $t>0$. And this gives by null-additivity,

$$
\mu\left(\left\{f \mathbb{1}_{E^{c}} \geq t\right\}\right)=\mu(\{f \geq t\})
$$

and we conclude by integrating on $[0, \infty), \int f d \mu=\int f \mathbb{1}_{E^{c}} d \mu$.

For the second equivalence, $(\Leftarrow)$ is straightforward with $g=f \mathbb{1}_{E^{c}}$. For $(\Rightarrow)$ we have, $\int f d \mu=\int f \mathbb{1}_{E^{c}} d \mu=\int g \mathbb{1}_{E^{c}} d \mu=\int g d \mu$.

The relationship between order continuity, null-additivity is thoroughly studied in [9] among other properties such as strong order continuity, exhaustivity or null-continuity.

\section{Convergence theorems}

\subsection{Almost everywhere convergence}

We will now focus on Property 2.3 and extend it to monotone a.e. convergence. $\mu$ is said to be strongly continuous from above if for all $E, F_{n}$ such that $\mu(E)=0$ and $F_{n} \downarrow F$ with $F_{n} \cap E=\emptyset$ then $\mu\left(E \cup F_{n}\right) \downarrow \mu(F)$.

In particular $\mu$ is strongly order continuous i.e., for all $A_{n} \downarrow A$ with $\mu(A)=0$ then $\mu\left(A_{n}\right) \downarrow 0$ (let $F_{n}=A_{n} \backslash A$ and $E=A$ ). By definition a strongly continuous from above set function is monotone (take $E=\emptyset, F_{1}=A, F_{n}=B$ for $n \geq 2$ ).

Proposition 3.1 Let $\mu$ be a set function. Then, $\mu$ is strongly continuous from above $\Longleftrightarrow \mu$ is continuous from above and null-additive.

Proof: $(\Rightarrow)$ From the definition of strong continuity from above with $E=\emptyset$ we obtain continuity from above.

With $F_{n}=F$ we obtain null-additivity.

$(\Leftarrow)$ Let $E, F_{n}$ be such that $\mu(E)=0$ and $F_{n} \downarrow F$ with $F_{n} \cap E=\emptyset$. We have $\mu\left(E \cup F_{n}\right) \downarrow \mu(E \cup F)$ by continuity and $\mu(E \cup F)=\mu(F)$ by null-additivity.

Theorem 3.1 Let $\mu$ be a set function. Then, $\mu$ is strongly continuous from above

$$
\Longleftrightarrow \forall f_{n}, f \in B_{\infty}(\Omega), f_{n} \downarrow_{a . e .} f \Rightarrow \int f_{n} d \mu \downarrow \int f d \mu
$$


Proof: Monotonicity is immediate.

$(\Leftarrow)$ Let $\mu(E)=0$ and $F_{n} \downarrow F$ with $F_{n} \cap E=\emptyset$. Then $\mathbb{1}_{E \cup F_{n}} \downarrow_{\text {a.e. }} \mathbb{1}_{F}$, thus $\mu\left(E \cup F_{n}\right) \downarrow \mu(F)$.

$(\Rightarrow)$ Since $\mu$ is finite, by constant additivity it suffices to prove the statement for $f_{n}, f \in B_{\infty}^{+}(\Omega)$.

Let $f_{n} \downarrow_{a . e .} f$. There exists $E$ with $\mu(E)=0$ such that $f_{n} \mathbb{1}_{E^{c}} \downarrow_{\text {e. }} f \mathbb{1}_{E^{c}}$. By the monotone convergence theorem for continuous from above set functions (see e.g. [10]) we have $\int f_{n} \mathbb{1}_{E^{c}} d \mu \downarrow \int f \mathbb{1}_{E^{c}} d \mu$.

Since $\mu(E)=0$ null-additivity entails $\int g d \mu=\int g \mathbb{1}_{E^{c}} d \mu$ for $g=f_{n}, f$. We get $\int f_{n} d \mu \downarrow \int f d \mu$.

A way to construct strongly continuous from above set functions is to take: $\mu=f_{o} P$ with $P$ a (non-atomic) $\sigma$-additive probability and $f:[0,1] \longrightarrow[0,1]$, non-decreasing, $f$ right-continuous with $f(p)>0$ for $p>0$.

Analogously we can provide some theorem with continuity from below. $\mu$ is said to be strongly continuous from below if for all $E, F_{n}$ such that $\mu(F)=0$ and $F_{n} \downarrow F$ with $F_{n} \subset E$ then $\mu\left(E \backslash F_{n}\right) \uparrow \mu(E)$.

Proposition 3.2 Let $\mu$ be a set function. $\mu$ is strongly continuous from below $\Longleftrightarrow \mu$ is continuous from below and null-additive.

Proof: $(\Rightarrow)$ To prove continuity from above, let $A_{n} \uparrow A$. Set $E=A, F_{n}=$ $A \backslash A_{n}$. We get $A \backslash A_{n} \downarrow \emptyset$ thus $\mu\left(A_{n}\right)=\mu\left(E \backslash F_{n}\right) \uparrow \mu(E)=\mu(A)$.

For $F_{n}=F$ we get null-additivity.

$(\Leftarrow)$ Let $E, F_{n}$ be such that $\mu(F)=0$ and $F_{n} \downarrow F$ with $F_{n} \subset E$. We have $\mu\left(E \backslash F_{n}\right) \uparrow \mu(E \backslash F)$ by continuity and $\mu(E \backslash F)=\mu(F)$ by null-additivity.

Theorem 3.2 Let $\mu$ be a set function. Then, $\mu$ is strongly continuous from below $\Longleftrightarrow$

$$
\forall f_{n}, f \in B_{\infty}(\Omega), f_{n} \uparrow_{a . e .} f \Rightarrow \int f_{n} d \mu \uparrow \int f d \mu
$$

Proof: Monotonicity is immediate.

$(\Leftarrow)$ Let $\mu(F)=0$ and $F_{n} \downarrow F$ with $F_{n} \subset E$. Then $\mathbb{1}_{E \backslash F_{n}} \uparrow_{\text {a.e. }} \mathbb{1}_{E}$, thus $\mu\left(E \backslash F_{n}\right) \uparrow$ $\mu(E)$.

$(\Rightarrow)$ Since $\mu$ is finite, by constant additivity it suffices to prove the statement for $f_{n}, f \in B_{\infty}^{+}(\Omega)$.

Let $f_{n} \uparrow_{\text {a.e. }} f$. There exists $E$ with $\mu(E)=0$ such that $f_{n} \mathbb{1}_{E^{c}} \uparrow_{e} . f \mathbb{1}_{E^{c}}$. By the monotone convergence theorem for continuous from below set functions (see e.g. [10]) we have $\int f_{n} \mathbb{1}_{E^{c}} d \mu \uparrow \int f \mathbb{1}_{E^{c}} d \mu$.

Since $\mu(E)=0$ null-additivity entails $\int g d \mu=\int g \mathbb{1}_{E^{c}} d \mu$ for $g=f_{n}, f$. We get $\int f_{n} d \mu \uparrow \int f d \mu$.

A combination of the previous theorems provides a Lebesgue's dominated convergence theorem. 
Theorem 3.3 Let $\mu$ be a set function.

Then $\mu$ is strongly continuous from below and above $\Longleftrightarrow$

$$
\forall f_{n}, f, g \in B_{\infty}(\Omega),\left|f_{n}\right| \leq g \text { a.e., } f_{n} \stackrel{\text { a.e. }}{\longrightarrow} f \Rightarrow \int f_{n} d \mu \rightarrow \int f d \mu, \int f d \mu \leq \int g d \mu
$$

Proof: $(\Rightarrow)$. We have $\mu\left(\left\{\left|f_{n}\right|>g\right\}\right)=0$ for all $n$. Thus by null-additivity $\mu\left(\cup_{i=1}^{n}\left\{\left|f_{i}\right|>g\right\}\right)=0$, and continuity from below entails $\mu\left(\cup_{n}\left\{\left|f_{n}\right|>g\right\}\right)=0$. Let $N=\cup_{n}\left\{\left|f_{n}\right|>g\right\}$. Consider the bounded measurable functions

$$
g_{n}=\sup _{k: k \geq n} f_{k} \mathbb{1}_{N^{c}}, h_{n}=\inf _{k: k \geq n} f_{k} \mathbb{1}_{N^{c}}
$$

we have $g_{n} \downarrow_{\text {a.e. }} f \mathbb{1}_{N^{c}}, h_{n} \uparrow_{\text {a.e. }} f \mathbb{1}_{N^{c}}$, applying Theorem $3.1,3.2$ gives us the conclusion.

$(\Leftarrow)$. We only check that $\mu$ is monotone. Take $f_{n}=f=\mathbb{1}_{A}$ and $g=\mathbb{1}_{B}$ with $A \subset B$. Then, $\mu(A)=\int f d \mu \leq \int g d \mu=\mu(B)$.

Remark 1 Since we deal with bounded measurable functions the condition of domination $\left[\forall n,\left|f_{n}\right| \leq g\right]$ can be restated as

$$
\exists M>0 / \forall n,\left|f_{n}\right| \leq M \cdot \mathbb{1}_{\Omega} .
$$

\subsection{Almost uniform convergence}

We will now extend Property 2.1 to monotone a.u. convergence. This type of convergence is related to the notion of autocontinuity which originates in Wang's 1984 founding article ([2]). As it will turn out, autocontinuity is related to a.u. convergence in the same manner than strong continuity is related to a.e. convergence.

Definition $3.1 \mu$ is autocontinuous from above if for all $E, F_{n}, E \cap F_{n}=\emptyset$, $\mu\left(F_{n}\right) \longrightarrow 0 \Rightarrow \mu\left(E \cup F_{n}\right) \longrightarrow \mu(E)$.

$\mu$ is autocontinuous from below if for all $E, F_{n}, F_{n} \subset E, \mu\left(F_{n}\right) \longrightarrow 0 \Rightarrow \mu(E \backslash$ $\left.F_{n}\right) \longrightarrow \mu(E)$.

\subsubsection{Monotone autocontinuity from above}

For our purpose we investigate a monotone version of autocontinuity one can guess already in Theorem 1 and 2 in [2] (see also [11]) and which is weaker than autocontinuity (see section 5),

Definition $3.2 \mu$ is monotone autocontinuous from above if for all $E, F_{n} \downarrow, E \cap$ $F_{n}=\emptyset, \mu\left(F_{n}\right) \downarrow 0 \Rightarrow \mu\left(E \cup F_{n}\right) \downarrow \mu(E)$. 
By definition a monotone autocontinuous from above set function is monotone (take $E=B, F_{1}=A \backslash B, F_{n}=\emptyset$ for $n \geq 2$ ) and null-additive (take $F_{n}=F$ and $\mu(F)=0)$.

We use the terminology of monotone autocontinuity from above, since we require that the sequence of $F_{n}$ 's must be non-increasing. This is a weaker version of strong continuity from above, for instance take $\mu$ additive but not $\sigma$-additive. The relation between monotone autocontinuity from above and strong continuity from above can be made more precise (see also Proposition 5 in [2], Theorem 5.8 in [12], Theorem 3.2 in [13]),

Proposition 3.3 Let $\mu$ be a set function. Then, $\mu$ is monotone autocontinuous from above and order continuous

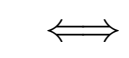

$\mu$ is strongly continuous from above.

Proof: $\quad(\Rightarrow)$. Let $E, F_{n} \downarrow F$ with $E \cap F_{n}=\emptyset$ and $\mu(E)=0$.

Since $F_{n} \backslash F \downarrow \emptyset$ we have $\mu\left(F_{n} \backslash F\right) \downarrow 0$ by order continuity. Monotone autocontinuity from above entails $\mu\left(F_{n}\right)=\mu\left(F_{n} \backslash F \cup F\right) \downarrow \mu(F)$. Now by null-additivity, $\mu\left(E \cup F_{n}\right)=\mu\left(F_{n}\right)$ for all $n$, thus $\mu\left(E \cup F_{n}\right) \downarrow \mu(F)$.

$(\Leftarrow)$.

To obtain order continuity take $E=F=\emptyset$ and $F_{n} \downarrow \emptyset$.

To obtain monotone autocontinuity from above take $E, F_{n} \downarrow, E \cap F_{n}=\emptyset$, with $\mu\left(F_{n}\right) \downarrow 0$. Put $F=\cap_{n} F_{n}$. We have $\mu(F)=0$ and so by continuity from above and null-additivity we get $\mu\left(E \cup F_{n}\right) \downarrow \mu(E \cup F)=\mu(E)$.

We may state now Property 2.1 for monotone a.u. convergence.

Theorem 3.4 Let $\mu$ be a set function.

Then $\mu$ is monotone autocontinuous from above $\Longleftrightarrow$

$$
\forall f_{n}, f \in B_{\infty}(\Omega), f_{n} \downarrow_{a . u .} f \Rightarrow \int f_{n} d \mu \downarrow \int f d \mu
$$

Proof: Monotonicity is immediate.

$(\Leftarrow)$ Let $E, F_{n} \downarrow, \mu\left(F_{n}\right) \downarrow 0$ and $\epsilon>0$. There exists $N_{\epsilon}$ such that $\mu\left(F_{N_{\epsilon}}\right)<\epsilon$. We have also for $n \geq N_{\epsilon}$,

$$
\mathbb{1}_{E \cup F_{n} \backslash F_{N_{\epsilon}}}=\mathbb{1}_{E \backslash F_{N_{\epsilon}}}=\mathbb{1}_{E \cup F \backslash F_{N_{\epsilon}}}
$$

so

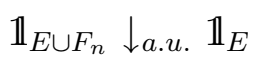

and by hypothesis $\mu\left(E \cup F_{n}\right)=\int \mathbb{1}_{E \cup F_{n}} d \mu \downarrow \int \mathbb{1}_{E} d \mu=\mu(E)$. 
$(\Rightarrow)$ Let $f_{n} \downarrow_{a . u .} f$. Without loss of generality we may assume that $0 \leq f_{n} \leq 1$ and that there exists a non-increasing sequence $E_{m} \supset E_{m+1}$ with $\mu\left(E_{m}\right)<\frac{1}{m}$ such that $f_{n} \mathbb{1}_{E_{m}^{c}} \downarrow_{u} f \mathbb{1}_{E_{m}^{c}}$.

Let $\epsilon>0$ and $m$ a positive integer. We have,

$$
\int f_{n} d \mu \leq \int f_{n} \mathbb{1}_{E_{m}^{c}}+\mathbb{1}_{E_{m}} d \mu \leq \int f \mathbb{1}_{E_{m}^{c}}+\mathbb{1}_{E_{m}}+\epsilon \mathbb{1}_{\Omega} d \mu
$$

for $n$ large enough since $f_{n} \mathbb{1}_{E_{m}^{c}}+\mathbb{1}_{E_{m}} \downarrow_{u} f \mathbb{1}_{E_{m}^{c}}+\mathbb{1}_{E_{m}}$. So,

$$
\lim _{n} \int f_{n} d \mu \leq \int f \mathbb{1}_{E_{m}^{c}}+\mathbb{1}_{E_{m}}+\epsilon \mathbb{1}_{\Omega} d \mu
$$

The right hand side gives,

$$
\begin{aligned}
& \int f \mathbb{1}_{E_{m}^{c}}+\mathbb{1}_{E_{m}}+\epsilon \mathbb{1}_{\Omega} d \mu \\
= & \int_{0}^{1} \mu\left(\left\{f \mathbb{1}_{E_{m}^{c}}+\mathbb{1}_{E_{m}}>t\right\}\right) d t+\epsilon \mu(\Omega) \\
= & \int_{0}^{1} \mu\left(\{f>t\} \cap E_{m}^{c} \cup E_{m}\right) d t+\epsilon \mu(\Omega) \\
= & \int_{0}^{1} \mu\left(\{f>t\} \cup E_{m}\right) d t+\epsilon \mu(\Omega)
\end{aligned}
$$

and since $\mu\left(\{f>t\} \cup E_{m}\right) \downarrow \mu(\{f>t\})$ for all $t \in(0,1)$ and $\mu\left(\{f>t\} \cup E_{1}\right) \leq$ $\mu(\Omega)$ the monotone convergence theorem concludes that

$$
\lim _{n} \int f_{n} d \mu \leq \int f d \mu+\epsilon \mu(\Omega)
$$

Analogously we can provide a theorem with continuity from below.

\subsubsection{Monotone autocontinuity from below}

Definition $3.3 \mu$ is monotone autocontinuous from below if for all $E, F_{n} \downarrow, F_{n} \subset$ $E, \mu\left(F_{n}\right) \downarrow 0 \Rightarrow \mu\left(E \backslash F_{n}\right) \uparrow \mu(E)$.

The relation between monotone autocontinuity from below and strong continuity from below can be made more precise (see Proposition 5 in [2], Theorem 3.3 in $[13])$,

Proposition 3.4 Let $\mu$ be a set function. If $\mu$ is strongly continuous from below then $\mu$ is monotone autocontinuous from below. The converse holds if $\mu$ is order continuous.

Proof: Let $F_{n} \downarrow F, F_{n} \subset E$ with $\mu\left(F_{n}\right) \downarrow 0$. Hence $\mu(F)=0$, thus $\mu\left(E \backslash F_{n}\right) \uparrow$ $\mu(E)$.

For the converse. Let $F_{n} \downarrow F, F_{n} \subset E$ with $\mu(F)=0$. By order continuity we have $\mu\left(F_{n} \backslash F\right) \downarrow 0$. And monotone autocontinuity from below entails $\mu((E \backslash F) \backslash$ $\left.\left(F_{n} \backslash F\right)\right) \uparrow \mu(E \backslash F)=\mu(E)$, by null-additivity. 
We can note that the converse can hold if we do not assume order continuity.

Example 1 ([14]) Let $P$ be a non-atomic $\sigma$-additive probability and consider the distortion function $f$ defined by $f(p)=1$ for $p \in(0,1], f(0)=0$. Let $\mu=$ foP. . We have

$$
\int f d \mu=\operatorname{ess}_{\mathrm{P}} \sup f=\inf \{M: M \in \mathbb{R} \text { such that } P(\{f>M\})=0\} .
$$

This set function is strongly continuous from below, monotone autocontinuous from above, but is not order continuous.

Theorem 3.5 Let $\mu$ be a set function. Then, $\mu$ is monotone autocontinuous from below $\Longleftrightarrow$

$$
\forall f_{n}, f \in B_{\infty}(\Omega), f_{n} \uparrow a . u . f \Rightarrow \int f_{n} d \mu \uparrow \int f d \mu
$$

Proof: Monotonicity is immediate.

$(\Leftarrow)$ Let $E, F_{n} \downarrow, \mu\left(F_{n}\right) \downarrow 0$ and $\epsilon>0$. There exists $N_{\epsilon}$ such that $\mu\left(F_{N_{\epsilon}}\right)<\epsilon$. We have also for $n \geq N_{\epsilon}$,

$$
\mathbb{1}_{\left(E \backslash F_{n}\right) \backslash F_{N_{\epsilon}}}=\mathbb{1}_{E \backslash F_{N_{\epsilon}}}
$$

SO

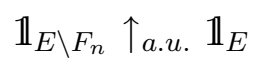

and by hypothesis $\mu\left(E \backslash F_{n}\right)=\int \mathbb{1}_{E \backslash F_{n}} d \mu \uparrow \int \mathbb{1}_{E} d \mu=\mu(E)$.

$(\Rightarrow)$ Let $f_{n} \uparrow_{\text {a.u. }} f$. Without loss of generality we may assume that $0 \leq f_{n} \leq 1$ and that there exists a non-increasing sequence $E_{m} \supset E_{m+1}$ with $\mu\left(E_{m}\right)<\frac{1}{m}$ such that $f_{n} \mathbb{1}_{E_{m}^{c}} \uparrow_{u} f \mathbb{1}_{E_{m}^{c}}$.

Assume there exists $c>0$ such that $c \leq f_{1}(\omega)$ for all $\omega \in \Omega$.

Let $\epsilon \in(0, c)$ and $m$ a positive integer. We have,

$$
\int f_{n} d \mu \geq \int f_{n} \mathbb{1}_{E_{m}^{c}} d \mu \geq \int f \mathbb{1}_{E_{m}^{c}}-\epsilon \mathbb{1}_{E_{m}^{c}} d \mu
$$

for $n$ large enough since $f_{n} \mathbb{1}_{E_{m}^{c}} \uparrow_{u} f \mathbb{1}_{E_{m}^{c}}$. So,

$$
\lim _{n} \int f_{n} d \mu \geq \int f \mathbb{1}_{E_{m}^{c}}-\epsilon \mathbb{1}_{E_{m}^{c}} d \mu
$$

The right hand side gives,

$$
\begin{aligned}
& \int f \mathbb{1}_{E_{m}^{c}}-\epsilon \mathbb{1}_{E_{m}^{c}} d \mu \\
= & \int f \mathbb{1}_{E_{m}^{c}} d \mu-\epsilon \mu\left(E_{m}^{c}\right), \text { by comonotonicity } \\
\geq & \int f \mathbb{1}_{E_{m}^{c}} d \mu-\epsilon \mu(\Omega) \\
= & \int_{0}^{1} \mu\left(\{f>t\} \backslash E_{m}\right) d t-\epsilon \mu(\Omega)
\end{aligned}
$$


and since $\mu\left(\{f>t\} \backslash E_{m}\right) \uparrow \mu(\{f>t\})$ for all $t \in(0,1)$ the monotone convergence theorem concludes that

$$
\lim _{n} \int f_{n} d \mu \geq \int f d \mu-\epsilon \mu(\Omega)
$$

Now if inf $f_{1}=0$. Set $\hat{g}=\frac{1}{2}\left(g+\mathbb{1}_{\Omega}\right)$ for $g=f, f_{n}$. We have $\frac{1}{2} \mathbb{1}_{\Omega} \leq \hat{f}_{n} \leq \mathbb{1}_{\Omega}$ and $\hat{f}_{n} \uparrow a . u$. $\hat{f}$. So $\lim _{n} \int \hat{f}_{n} d \mu \uparrow \int \hat{f} d \mu$ and by comonotonic additivity we get $\lim _{n} \int f_{n} d \mu \uparrow \int f d \mu$.

\subsubsection{Monotone autocontinuity}

A combination of the previous theorems gives us a Lebesgue's type dominated convergence theorem without sequential continuity.

Theorem 3.6 Let $\mu$ be a set function. Then, $\mu$ is monotone autocontinuous $\Longleftrightarrow$

$$
\forall f_{n}, f, g \in B_{\infty}(\Omega),\left|f_{n}\right| \leq g, f_{n} \stackrel{a . u .}{\longrightarrow} f \Rightarrow \int f_{n} d \mu \rightarrow \int f d \mu, \int f d \mu \leq \int g d \mu
$$

Proof: The same as in the classical case. Consider the bounded measurable functions

$$
g_{n}=\sup _{k: k \geq n} f_{k}, h_{n}=\inf _{k: k \geq n} f_{k}
$$

We can check that $g_{n}, h_{n}$ are $\downarrow_{a . u .}, \uparrow_{a . u .} f$ and $-g \leq h_{n} \leq g_{n} \leq g$.

Since $f_{n} \stackrel{a . u .}{\longrightarrow} f$ there are $E_{m} \supset E_{m+1}$ with $\mu\left(E_{m}\right)<\frac{1}{m}$ such that $f_{n} \mathbb{1}_{E_{m}^{c}} \stackrel{u .}{\longrightarrow}$ $f \mathbb{1}_{E_{m}^{c}}$. Let $p$ be a positive integer. There exists $N_{p}$ such that for all $n \geq N_{p}$ and for all $\omega \in E_{m}^{c}$,

$$
f(\omega)-\epsilon \leq f_{n}(\omega) \leq f(\omega)+\epsilon
$$

thus

$$
f(\omega)-\epsilon \leq h_{N_{p}}(\omega) \leq g_{N_{p}}(\omega) \leq f(\omega)+\epsilon .
$$

So $h_{N_{p}} \uparrow_{a . u .} f, g_{N_{p}} \downarrow_{\text {a.u. }} f$ and $h_{n} \uparrow_{\text {a.u. }} f, g_{n} \downarrow_{\text {a.u. }} f$ follow.

Remark 2 The domination condition in Theorem 3.6 can be stated in a more general manner as, $\left[\forall n,\left|f_{n}\right| \leq g\right]$ almost everywhere, i.e.,

$$
\mu\left(\cup_{n}\left\{\left|f_{n}\right|>g\right\}\right)=0 .
$$

If $\mu$ is $\sigma$-null-additive the domination condition reduces to for all $n,\left[\left|f_{n}\right| \leq g\right]$ almost everywhere, i.e.,

$$
\forall n, \mu\left(\left\{\left|f_{n}\right|>g\right\}\right)=0 .
$$


We can state a sufficient condition for an Egoroff's theorem $([15,16]$, see also [17] for a Lebesgue's Theorem).

Corollary 3.1 Let $\mu$ be a monotone autocontinuous set function. Then, $\mu$ is order continuous $\Longleftrightarrow$

$$
\forall f_{n}, f \in B_{\infty}(\Omega), f_{n} \stackrel{\text { a.e. }}{\longrightarrow} f \Rightarrow f_{n} \stackrel{a . u .}{\longrightarrow} f
$$

Proof: $(\Rightarrow)$ From Propositions 3.3, 3.4 and Corollary 6.4 in [12].

$(\Leftarrow)$ Since $\mu$ is monotone autocontinuous, for any sequence $\left\{f_{n}\right\}_{n}$ with $f_{n} \stackrel{\text { a.e. }}{\longrightarrow} f$ and $\left|f_{n}\right|,|f| \leq|g|$ we have $\int f_{n} d \mu \rightarrow \int f d \mu$, hence the sufficient part in Theorem 3.3 entails order continuity.

\subsection{Convergence in measure}

A sequence $\left\{f_{n}\right\}_{n}$ of bounded functions is said to converge strictly in measure to $f$ denoted by $f_{n} \stackrel{s-\mu}{\longrightarrow} f$ if $\lim _{n} \mu\left(\left\{\left|f_{n}-f\right|>0\right\}\right)=0$. The $s-\mu$ convergence is stronger than convergence in measure which is defined as follows

$$
f_{n} \stackrel{\mu}{\longrightarrow} f \text { if } \forall \epsilon>0, \lim _{n} \mu\left(\left\{\left|f_{n}-f\right| \geq \epsilon\right\}\right)=0
$$

Similarly convergence in measure is stronger than mean-convergence (see Theorem 2 in [3]), i.e.,

$$
f_{n} \stackrel{m}{\longrightarrow} f \text { if } \lim _{n} \int\left|f_{n}-f\right| \mu=0 .
$$

If we assume that the domination condition holds i.e. $\left|f_{n}\right|,|f| \leq g, \in B_{\infty}(\Omega, \mathcal{A})$ then $f_{n} \stackrel{\mu}{\longrightarrow} f \Rightarrow f_{n} \stackrel{m}{\longrightarrow} f$ and a fortiori $f_{n} \stackrel{s-\mu}{\longrightarrow} f \Rightarrow f_{n} \stackrel{m}{\longrightarrow} f$.

These $s-\mu, m, \mu$ convergences fully characterize autocontinuity. In particular, the equivalence $(i) \Longleftrightarrow$ (ii) with $s-\mu$ convergence can be an alternative to Theorem 3.6 with a.u. convergence.

Theorem 3.7 Let $\mu$ be a monotone set function. The following assertions are equivalent,

(i) $\mu$ is autocontinuous,

(ii) $\forall f_{n}, f, g \in B_{\infty}(\Omega),\left|f_{n}\right|,|f| \leq g, f_{n} \stackrel{s-\mu}{\longrightarrow} f \Rightarrow \int f_{n} d \mu \longrightarrow \int f d \mu$,

(iii) $\forall f_{n}, f, g \in B_{\infty}(\Omega),\left|f_{n}\right|,|f| \leq g, f_{n} \stackrel{\mu}{\longrightarrow} f \Rightarrow \int f_{n} d \mu \longrightarrow \int f d \mu$,

(iv) $\forall f_{n}, f, g \in B_{\infty}(\Omega),\left|f_{n}\right|,|f| \leq g, f_{n} \stackrel{m}{\longrightarrow} f \Rightarrow \int f_{n} d \mu \longrightarrow \int f d \mu$.

The equivalence of $(i),($ iii $)$ is a restatement of Theorem 6 and 7 in [3] where the condition of equiintegrability is dropped and the local uniform autocontinuity or the continuity conditions become too strong. Other formulations can be found as Theorem 3.5 in [18] and as Theorem 3.3 in [16]. 
Proof: $\quad(i i) \Rightarrow(i)$. Let $E, F_{n}, E \cap F_{n}=\emptyset$ and set $f_{n}=\mathbb{1}_{E \cup F_{n}}, f=\mathbb{1}_{E}, g=\mathbb{1}_{\Omega}$. Assume $\mu\left(F_{n}\right) \downarrow 0$. That is, $\mu\left(\left\{\left|f_{n}-f\right|>0\right\}\right) \downarrow 0$. We get $\mu\left(E \cup F_{n}\right)=\int f_{n} d \mu \longrightarrow$ $\int f d \mu=\mu(E)$.

Similarly, let $E, F_{n}, F_{n} \subset E$ with $\mu\left(F_{n}\right) \downarrow 0$ and set $f_{n}=\mathbb{1}_{E \backslash F_{n}}, f=\mathbb{1}_{E}, g=\mathbb{1}_{\Omega}$. $(i i i) \Rightarrow(i),(i v) \Rightarrow(i)$. Same as $(i i) \Rightarrow(i)$.

$(i) \Rightarrow(i i)$. Since $\mu$ is assumed to be autocontinuous we can assume that $\left|f_{n}\right|,|f| \leq g$. By constant additivity we only consider the case where $f_{n}, f \geq 0$. Let $t \in[0, M]$ where $M=\sup g$. We have,

$$
\left\{f_{n} \geq t\right\} \subset\{f \geq t\} \cup\left\{\left|f_{n}-f\right|>0\right\}
$$

thus

$$
\mu\left(\left\{f_{n} \geq t\right\}\right) \leq \mu\left(\{f \geq t\} \cup\left\{\left|f_{n}-f\right|>0\right\}\right) \leq \mu(\Omega)
$$

and by integration,

$$
\int f_{n} d \mu \leq \int_{[0, M]} \mu\left(\{f \geq t\} \cup\left\{\left|f_{n}-f\right|>0\right\}\right) d \lambda(t) .
$$

Now since $\mu$ is autocontinuous from above we also have

$$
\mu\left(\{f \geq t\} \cup\left\{\left|f_{n}-f\right|>0\right\}\right) \longrightarrow \mu(\{f \geq t\}),
$$

thus by the dominated convergence theorem we get

$$
\varlimsup \int f_{n} d \mu \leq \int_{[0, M]} \mu(\{f \geq t\}) d \lambda(t)=\int f d \mu .
$$

Similarly, since

$$
\{f \geq t\} \backslash\left\{\left|f_{n}-f\right|>0\right\} \subset\left\{f_{n} \geq t\right\}
$$

thus

$$
\mu\left(\{f \geq t\} \backslash\left\{\left|f_{n}-f\right|>0\right\}\right) \leq \mu\left(\left\{f_{n} \geq t\right\}\right) \leq \mu(\Omega)
$$

and by integration,

$$
\int_{[0, M]} \mu\left(\{f \geq t\} \backslash\left\{\left|f_{n}-f\right|>0\right\}\right) d \lambda(t) \leq \int f_{n} d \mu .
$$

Now since $\mu$ is autocontinuous from below we also have

$$
\mu\left(\{f \geq t\} \backslash\left\{\left|f_{n}-f\right|>0\right\}\right) \longrightarrow \mu(\{f \geq t\}),
$$

thus by the dominated convergence theorem we get

$$
\int f d \mu=\int_{[0, M]} \mu(\{f \geq t\}) d \lambda(t) \leq \underline{\lim } \int f_{n} d \mu .
$$

Since $\epsilon$ is arbitrary, we obtain $\int f d \mu=\lim _{n} \int f_{n} d \mu$.

$(i) \Rightarrow(i i i)$. The proof is adapted from [3].

Since $\mu$ is assumed to be autocontinuous we can assume that $\left|f_{n}\right|,|f| \leq g$. By 
constant additivity we only consider the case where $f_{n}, f \geq 0$.

Let $t \in[0, M]$ where $M=\sup g$ and $\epsilon>0$. We have,

$$
\left\{f_{n} \geq t+\epsilon\right\} \subset\{f \geq t\} \cup\left\{\left|f_{n}-f\right| \geq \epsilon\right\}
$$

thus

$$
\mu\left(\left\{f_{n} \geq t+\epsilon\right\}\right) \leq \mu\left(\{f \geq t\} \cup\left\{\left|f_{n}-f\right| \geq \epsilon\right\}\right) \leq \mu(\Omega)
$$

and by integration,

$\int f_{n} d \mu-\epsilon \mu(\Omega) \leq \int_{[\epsilon, M]} \mu\left(\left\{f_{n} \geq t\right\}\right) d \lambda(t) \leq \int_{[0, M]} \mu\left(\{f \geq t\} \cup\left\{\left|f_{n}-f\right| \geq \epsilon\right\}\right) d \lambda(t)$.

Now since $\mu$ is autocontinuous from above we also have

$$
\mu\left(\{f \geq t\} \cup\left\{\left|f_{n}-f\right| \geq \epsilon\right\}\right) \longrightarrow \mu(\{f \geq t\}),
$$

thus by the dominated convergence theorem we get

$$
\varlimsup f_{n} d \mu-\epsilon \mu(\Omega) \leq \int_{[0, M]} \mu(\{f \geq t\}) d \lambda(t)=\int f d \mu .
$$

Similarly, since

$$
\{f \geq t\} \backslash\left\{\left|f_{n}-f\right| \geq \epsilon\right\} \subset\left\{f_{n} \geq t-\epsilon\right\}
$$

thus

$$
\mu\left(\{f \geq t\} \backslash\left\{\left|f_{n}-f\right| \geq \epsilon\right\}\right) \leq \mu\left(\left\{f_{n} \geq t-\epsilon\right\}\right) \leq \mu(\Omega)
$$

and by integration,

$$
\begin{aligned}
& \int_{[0, M]} \mu\left(\{f \geq t\} \backslash\left\{\left|f_{n}-f\right| \geq \epsilon\right\}\right) d \lambda(t) \\
\leq & \epsilon \mu(\Omega)+\int_{[\epsilon, M]} \mu\left(\{f \geq t\} \backslash\left\{\left|f_{n}-f\right| \geq \epsilon\right\}\right) d \lambda(t) \\
\leq & \epsilon \mu(\Omega)+\int_{[\epsilon, M]} \mu\left(\left\{f_{n} \geq t-\epsilon\right\}\right) d \lambda(t) \\
\leq & \epsilon \mu(\Omega)+\int_{[0, M]} \mu\left(\left\{f_{n} \geq t\right\}\right) d \lambda(t) \\
= & \epsilon \mu(\Omega)+\int f_{n} d \mu
\end{aligned}
$$

Now since $\mu$ is autocontinuous from below we also have

$$
\mu\left(\{f \geq t\} \backslash\left\{\left|f_{n}-f\right| \geq \epsilon\right\}\right) \longrightarrow \mu(\{f \geq t\}),
$$

thus by the dominated convergence theorem we get

$$
\int f d \mu=\int_{[0, M]} \mu(\{f \geq t\}) d \lambda(t) \leq \underline{\lim } \int f_{n} d \mu+\epsilon \mu(\Omega) .
$$

Since $\epsilon$ is arbitrary, we obtain $\int f d \mu=\lim _{n} \int f_{n} d \mu$. 
$($ iii $) \Rightarrow(i v)$. Using Markov's inequality it holds, for all $\epsilon>0$,

$$
\epsilon \cdot \mu\left(\left\{\left|f_{n}-f\right| \geq \epsilon\right\}\right) \leq \int\left|f_{n}-f\right| d \mu \longrightarrow 0 .
$$

We add a direct proof of $(i v) \Rightarrow(i i i)$. Let $\sup g=T<\infty$. Then, for all $t \in[0, T]$

$$
\mu\left(\left\{\left|f_{n}-f\right| \geq t\right\}\right) \leq \mu\left(\left\{\left|f_{n}\right| \geq t / 2\right\} \cup\{|f| \geq t / 2\}\right) \leq \mu(\{g \geq t / 2\})
$$

and $\int_{0}^{T} \mu(\{g \geq t / 2\}) d t=2 \int g d \mu<\infty$.

Assume $f_{n} \stackrel{\mu}{\longrightarrow} f$, then by the dominated convergence theorem applied to the decumulative function $\mu\left(\left\{\left|f_{n}-f\right| \geq().\right\}\right)$ on $[0, T]$ it comes

$$
\int\left|f_{n}-f\right| d \mu=\int_{0}^{T} \mu\left(\left\{\left|f_{n}-f\right| \geq t\right\}\right) d t \longrightarrow 0
$$

that is, $f_{n} \stackrel{m}{\longrightarrow} f$.

Remark 3 The domination condition in Theorem 3.7 can be stated to hold almost everywhere (see Remark 2).

We may extract from the proof the following theorems, where $s-\mu$ convergence can either be replaced by $\mu$ or $m$ convergence,

Theorem 3.8 Let $\mu$ be a monotone set function. Then, $\mu$ is autocontinuous from above $\Longleftrightarrow$

$$
\forall f_{n}, f, g \in B_{\infty}(\Omega),\left[\left|f_{n}\right|,|f| \leq g\right] \text { a.e., } f_{n} \stackrel{s-\mu}{\longrightarrow} f \Rightarrow \varlimsup \int f_{n} d \mu \leq \int f d \mu .
$$

Theorem 3.9 Let $\mu$ be a monotone set function. Then, $\mu$ is autocontinuous from below $\Longleftrightarrow$

$$
\forall f_{n}, f, g \in B_{\infty}(\Omega),\left[\left|f_{n}\right|,|f| \leq g\right] \text { a.e., } f_{n} \stackrel{s-\mu}{\longrightarrow} f \Rightarrow \int f d \mu \leq \underline{\lim } \int f_{n} d \mu .
$$

Strict convergence in measure is neither weaker nor stronger than a.e. convergence, a.u. convergence or $m$ convergence. The domination condition is necessary to prove that $\mu$ convergence implies $m$ convergence.

Example 2 Let $\mu$ be the Lebesgue measure on $\Omega=[0,1)$ endowed with its Borel $\sigma$-algebra. Define the following sequences,

$$
\begin{gathered}
f_{n}=\frac{1}{n} \mathbb{1}_{[0,1)}, \\
g_{n}=\mathbb{1}_{\left[\frac{p}{2^{m}}, \frac{p+1}{2^{m}}\right)} \text { for } n=1+\ldots+2^{m-1}+p, p=0, \ldots, 2^{m}-1,
\end{gathered}
$$




$$
h_{n}=n \mathbb{1}_{\left(0, \frac{1}{n}\right)} .
$$

Then,

$$
\begin{array}{ll}
f_{n} \stackrel{\text { a.u. }}{\longrightarrow} \mathbb{1}_{\emptyset} & \text { and } f_{n} \stackrel{s-\mu}{\nrightarrow} \mathbb{1}_{\emptyset} \\
g_{n} \stackrel{s-\mu}{\longrightarrow} \mathbb{1}_{\emptyset} & \text { and } g_{n} \stackrel{\text { a.u. }}{\nrightarrow} \mathbb{1}_{\emptyset}, g_{n} \stackrel{\text { a.e. }}{\nrightarrow} \mathbb{1}_{\emptyset} \\
h_{n} \stackrel{s-\mu}{\longrightarrow} \mathbb{1}_{\emptyset} & \text { and } h_{n} \stackrel{m}{\rightarrow} \mathbb{1}_{\emptyset} .
\end{array}
$$

\section{Dual Results}

Our previous results dealing with a.e. and a.u. convergence can be translated with pseudo convergence. A sequence $\left\{f_{n}\right\}_{n}$ is said to pseudo everywhere converge to $f$ if there is a set $E$ with $\mu(E)=\mu(\Omega)$ such that $\left\{f_{n} \mathbb{1}_{E}\right\}_{n}$ converges to $f \mathbb{1}_{E}$ (see [20]). Similarly, $\left\{f_{n}\right\}_{n}$ is said to pseudo converge uniformly to $f$ if for all $\epsilon>0$ there is a set $E_{\epsilon}$ with $\mu\left(E_{\epsilon}\right)>\mu(\Omega)-\epsilon$ such that $\left\{f_{n} \mathbb{1}_{E_{\epsilon}}\right\}_{n}$ converges uniformly to $f \mathbb{1}_{E_{\epsilon}}$. Otherwise stated pseudo-convergence with respect to $\mu$ is convergence with respect to $\bar{\mu}$.

For this reason we introduce the converse definition of the various continuity definition related to the conjugate set functions. A set function $\mu$ is said to be, pseudo strongly continuous from above if for all $G, H_{n} \uparrow H, H_{n} \supset G$, with $\mu(H)=$ $\mu(\Omega)$ then $\mu\left(G \cup H_{n}^{c}\right) \downarrow \mu(G)$,

pseudo strongly continuous from below if for all $G, H_{n} \uparrow H, G \cup H_{n}=\Omega$, with $\mu(H)=\mu(\Omega)$ then $\mu\left(G \cap H_{n}\right) \uparrow \mu(G)$,

pseudo monotone autocontinuous from above if for all $G, H_{n} \uparrow, H_{n} \supset G, \mu\left(H_{n}\right) \uparrow$ $\mu(\Omega)$ then $\mu\left(G \cup H_{n}^{c}\right) \downarrow \mu(G)$,

pseudo monotone autocontinuous from below if for all $G, H_{n} \uparrow, G \cup H_{n}=\Omega$, $\mu\left(H_{n}\right) \uparrow \mu(\Omega)$ then $\mu\left(G \cap H_{n}\right) \uparrow \mu(G)$.

These definitions are simply restating that $\bar{\mu}$ is respectively strongly continuous from below, strongly continuous from above, monotone autocontinuous from below and monotone autocontinuous from above. We can now formulate the pseudo-convergence theorems.

Corollary 4.1 Let $\mu$ be a set function. Then, $\mu$ is pseudo strongly continuous from above $\Longleftrightarrow$

$$
\forall f_{n}, f \in B_{\infty}(\Omega), f_{n} \downarrow_{\text {p.a.e. }} f \Rightarrow \int f_{n} d \mu \downarrow \int f d \mu
$$

Corollary 4.2 Let $\mu$ be a set function. Then, $\mu$ is pseudo strongly continuous from below $\Longleftrightarrow$

$$
\forall f_{n}, f \in B_{\infty}(\Omega), f_{n} \uparrow_{p . a . e .} f \Rightarrow \int f_{n} d \mu \uparrow \int f d \mu
$$


Corollary 4.3 Let $\mu$ be a set function. Then, $\mu$ is pseudo monotone autocontinuous from above $\Longleftrightarrow$

$$
\forall f_{n}, f \in B_{\infty}(\Omega), f_{n} \downarrow_{\text {p.a.u. }} f \Rightarrow \int f_{n} d \mu \downarrow \int f d \mu
$$

Corollary 4.4 Let $\mu$ be a set function. Then, $\mu$ is pseudo monotone autocontinuous from below $\Longleftrightarrow$

$$
\forall f_{n}, f \in B_{\infty}(\Omega), f_{n} \uparrow_{p . a . u .} f \Rightarrow \int f_{n} d \mu \uparrow \int f d \mu
$$

Proof: The proofs rely on the duality formula, i.e., $\int f d \bar{\mu}=-\int-f d \mu$, the fact that $f_{n} \downarrow_{\text {p.a. }} f \Longleftrightarrow-f_{n} \uparrow_{\text {p.a. }}-f$ and that pseudo almost convergence with respect to $\mu$ is equivalent to almost convergence with respect to $\bar{\mu}$.

Example 3 ([14]) The essential infimum functional, defined as

$$
\operatorname{ess}_{\mathrm{P}} \inf f=\sup \{M: M \in \mathbb{R} \text { such that } P(\{f>M\})=1\}
$$

is continuous with respect to pseudo almost uniform convergence but not with respect to pseudo almost everywhere convergence.

\section{Counterexamples}

We shall present four examples of set functions which clarify the relationship between autocontinuity from above (below), monotone autocontinuity from above (below) and order continuity (see also [9, 11, 21]).

Examples 4 and 5 show that monotone autocontinuity and order continuity are independent properties. Example 5 show that monotone autocontinuity from above (below) is weaker than autocontinuity from above (below) even under order continuity. Examples 6 and 7 show that monotone autocontinuity from above and from below are independent even under order continuity.

These examples are given on $\mathbb{N}=\{1,2,3, \ldots\}$ the set of positive integers. On $\mathbb{N}$, order continuity can be characterized ${ }^{2}$ in a simple way by the necessary condition

$$
\lim _{n \longrightarrow \infty} \mu([n, \infty))=0
$$

Example 4 Let $\Omega=\mathbb{N}$ and $\mathcal{A}=2^{\mathrm{N}}$. Define,

$$
\mu(A)= \begin{cases}1 /(4 n) & , \text { if } A=\{n\} \text { for some } n \\ \frac{1}{2 \min A} & , \text { if }|A|>1 \\ 0 & , \text { if } A=\emptyset\end{cases}
$$

Then, $\mu$ is neiher monotone autocontinuous from above nor from below but $\mu$ is order continuous.

\footnotetext{
${ }^{2}$ Indeed, let $A_{n} \downarrow \emptyset$. Then, $\min A_{n} \uparrow \infty$. Thus, $\mu\left(A_{n}\right) \leq \mu\left(\left[\min A_{n}, \infty\right)\right) \downarrow 0$.
} 
Proof: Let $n>1$. We have, $\mu([n, \infty))=1 /(2 n) \longrightarrow 0$ so $\mu$ is order continuous. But,

$$
\mu(\{1\} \cup[n, \infty))=1 / 2 \nrightarrow 1 / 4=\mu(\{1\})
$$

and

$$
\mu([1, n-1])=1 / 2 \nrightarrow 1=\mu(\mathbb{N})
$$

so $\mu$ is not monotone autocontinuous from above and not monotone autocontinuous from below.

Example 5 Let $\Omega=\mathbb{N}$ and $\mathcal{A}=2^{\mathrm{N}}$. Define,

$$
\mu(A)= \begin{cases}1 /(4 n) & , \text { if } A=\{n\} \text { for some } n \\ 1 / 2 & , \text { if }|A|>1 \\ 0 & , \text { if } A=\emptyset\end{cases}
$$

Then, $\mu$ is monotone autocontinuous from above and below but $\mu$ is not order continuous and is neither autocontinuous from above nor from below.

Proof: $\mu$ is not order continuous since for all $n, \mu([n, \infty))=1 / 2$.

We check now that $\mu$ is monotone autocontinuous from above and below. Let $\left\{F_{n}\right\}_{n}$ be a decreasing sequence. Since each must $F_{n}$ is infinite, it holds for all $n, \mu\left(F_{n}\right) \geq 1 / 2$. Hence $\mu\left(F_{n}\right) \nrightarrow 0$, thus $\mu$ is monotone autocontinuous.

However, $\mu$ is not autocontinuous from above neither from below. Let $n>1$. We have,

$$
\mu(\{n\})=1 /(2 n) \longrightarrow 0
$$

but

$$
\mu(\{1, n\})=1 / 2 \nrightarrow 1 / 4=\mu(\{1\})
$$

and

$$
\mu(\mathbb{N} \backslash\{n\})=1 / 2 \nrightarrow 1=\mu(\mathbb{N}) .
$$

Example 6 Let $\Omega=\mathbb{N}$ and $\mathcal{A}=2^{\mathrm{N}}$. Define,

$$
\mu(A)= \begin{cases}1 /(2 n) & , \text { if } A=\{n\} \text { for some } n \\ 1 / \min A & , \text { if }|A|>1 \\ 0 & , \text { if } A=\emptyset\end{cases}
$$

Then, $\mu$ is not monotone autocontinuous from above but is monotone autocontinuous from below and order continuous.

Proof: Let $n>1$. We have,

$$
\mu([n, \infty))=1 / n \longrightarrow 0
$$


so $\mu$ is order continuous but $\mu$ is not monotone autocontinuous from above since

$$
\mu(\{1\} \cup[n, \infty))=1 \nrightarrow 1 / 2=\mu(\{1\})
$$

In order to prove monotone autocontinuity from below we shall prove the even stronger statement that $\mu$ is strongly continuous from below.

By Proposition 3.2, it suffices to prove that $\mu$ is null-additive and continuous from below. Since $\mu(A)>0$, whenever $A \neq \emptyset, \mu$ is null-additive.

Let us prove now that $\mu$ is continuous from below. Let $\left\{A_{n}\right\}_{n}$ be an increasing sequence to $A$. We may assume that $A$ is infinite. Put $\alpha=\min A$. Since $\left\{A_{n}\right\}_{n}$ is increasing to $A$ and $\alpha \in A$ thus there exists some $n(A)$ such that $\alpha \in A_{n(A)}$, hence

But $A_{n} \subset A$, so

$$
\min A_{n(A)} \leq \alpha
$$

$$
\min A_{n} \geq \alpha .
$$

For $n=\alpha$ we obtain, $\min A_{n(A)}=\alpha$. It follows that for $n \geq n(A)$,

$$
\alpha=\min A_{n(A)} \geq \min A_{n} \geq \alpha
$$

thus $\mu\left(A_{n}\right)=\mu(A)$.

Example 7 Let $\Omega=\mathbb{N}$ and $\mathcal{A}=2^{\mathrm{N}}$. Define,

$$
\mu(A)= \begin{cases}1 / \min A & , \text { if }|A|=\infty \\ 1 /(2 \min A) & , \text { if }|A|<\infty \\ 0 & , \text { if } A=\emptyset\end{cases}
$$

Then, $\mu$ is monotone autocontinuous from above and order continuous but is not monotone autocontinuous from below and is not autocontinuous from above.

Proof: Let $n>1$. We have, $\mu([n, \infty))=1 / n \longrightarrow 0$ so $\mu$ is order continuous. But $\mu$ is not monotone autocontinuous from below since

$$
\mu([1, n])=1 / 2 \nrightarrow 1=\mu(\mathbb{N}) .
$$

And $\mu$ is not autocontinuous from above, since

$$
\mu(\{1\} \cup[n, \infty))=1 \nrightarrow 1 / 2=\mu(\{1\}) .
$$

Let us prove that $\mu$ is monotone autocontinuous from above. Let $E, F_{n} \subset \mathbb{N}$ with $F_{n} \downarrow, \mu\left(F_{n}\right) \downarrow 0$ and $F_{n} \subset E$. Since $F_{n}$ is decreasing, each $F_{n}$ is infinite. As $\mu\left(F_{n}\right) \downarrow 0$, we have $\min F_{n} \uparrow \infty$, thus $F_{n} \downarrow \emptyset$.

Put $e=\min E$. Since $F_{n} \downarrow \emptyset$ there exists $n_{E}$ such that for $n \geq n_{E}$,

$$
F_{n} \cap[1, e]=\emptyset,
$$

thus

$$
\min F_{n}>e
$$

So we have for $n \geq n_{E}$,

$$
\min E \cup F_{n}=\min \left\{\min E, \min F_{n}\right\}=e
$$

thus $\mu\left(E \cup F_{n}\right)=\mu(E)$. 


\section{References}

[1] E. Pap. Null-additive set functions. Kluwer Academic Publishers, 1995.

[2] Z. Wang, The autocontinuity of set function and the fuzzy integral, Journal of Mathematical Analysis and Applications 99 (1984) 195-218.

[3] Z. Wang, Convergence theorems for sequences of Choquet integrals, International Journal of General Systems 26 (1997) 133-143.

[4] G. Choquet, Théorie des capacités, in: Annales de l'Institut Fourier '5, Grenoble, 1953-1954, 131-295.

[5] D. Denneberg, Non-additive measure and integral, Kluwer Acad. Publ. (1994).

[6] T. Murofushi and M. Sugeno, An interpretation of fuzzy measures and the Choquet integral as an integral with respect to a fuzzy measure, Fuzzy Sets and Systems 29 (1989) 201-227.

[7] D. Schmeidler, Integral representation without additivity, Proceedings of the American Mathematical Society 97 (1986) 255-261.

[8] T. Murofushi and M. Sugeno, A Theory of Fuzzy Measures: Representations, the Choquet Integral, and Null Sets, Journal of Mathematical Analysis and Applications 159 (1991) 532-549.

[9] S. Asahina, K. Uchino, T. Murofushi, Relationship among continuity conditions and null-additivity conditions in non-additive measure theory, Fuzzy sets and systems 157 (2006) 691-698

[10] Y. Rébillé, Sequentially continuous non-monotonic Choquet integrals, Fuzzy Sets and Systems 153 (2005) 79-94.

[11] M. Takahashi, S. Asahina and T. Murofushi, Conditions for convergence theorems in non-additive measure theory, RIMS Kokyuroku 1452 (2005) $11-21$.

[12] Z. Wang and G.J. Klir. Fuzzy Measure Theory. Plenum Press, New York, 1992.

[13] E. Pap, $\sigma$-null-additive set functions, Novi Sad Journal of Mathematics 32 (2002) 47-57.

[14] T. Murofushi and M. Sugeno, Some quantities represented by the Choquet integral, Fuzzy Sets and Systems 56 (1993) 229-235.

[15] J. Li, A further investigation for Egoroff's theorem with respect to monotone set functions, Kybernetika 39 (2003) 753-760. 
[16] T. Murofushi, K. Uchino, S. Asahina, Conditions for Egoroff's theorem in non-additive measure theory, Fuzzy Sets and Systems 146 (2004) 135-146.

[17] J. Li, Order continuous of monotone set function and convergence of measurable functions sequence, Applied Mathematics and Computation 135 (2003) 211-218.

[18] Dug Hun Hong, Kyung Tae Kim, Convergence of Choquet Integral, Journal of Applied Mathematics and Computing 18 (2005) 613-619.

[19] T. Murofushi, M. Sugeno and M. Suzaki, Autocontinuity, convergence in measure and convergence in distribution, Fuzzy Sets and Systems 92 (1997) 197-203.

[20] Z. Wang, Asymptotic structural characteristics of fuzzy measure and their applications, Fuzzy Sets and Systems 16 (1985) 277-290.

[21] Z. Wang, On the null-additivity and the autocontinuity of a fuzzy measure, Fuzzy Sets and Systems 45 (1992) 223-226. 